\title{
Effects of hadronic rescattering in mini-jet production and energy loss at the LHC
}

\section{Sangwook Ryu ${ }^{\star a b}$, Scott McDonald ${ }^{a}$, Chun Shen ${ }^{c}$, Sangyong Jeon ${ }^{a}$ and Charles Gale $^{a}$}

a Department of Physics, McGill University 3600 rue University, Montreal, Quebec H3A 2T8, Canada

$b$ Institut für Theoretische Physik, Goethe-Universität Frankfurt (FIAS)

Max-von-Laue-Str. 1, 60438 Frankfurt am Main, Germany

c Physics Department, Brookhaven National Laboratory

Upton, NY 11973, United States

E-mail: ryu@fias.uni-frankfurt.de, mcscottephysics.mcgill.ca,

chunshen1987@gmail.com, jeon@physics.mcgill.ca,

gale@physics.mcgill.ca

We present an extension of hybrid approach, which combines viscous hydrodynamics, particlization, jet energy loss and hadronic cascade, to describe data in the intermediate and high $p_{T}$ regimes. This enables us to study mini-jet production and energy loss in heavy ion collisions, in conjunction with the bulk dynamics of QGP fireball. This hybrid approach is applied for $\mathrm{Pb}-\mathrm{Pb}$ collisions at the LHC with $\sqrt{s_{N N}}=2.76 \mathrm{TeV}$, and we demonstrate that hadronic re-scatterings have significant effects on mini-jets with intermediate $p_{T}$.

12th International Workshop on High-pT Physics in the RHIC/LHC Era 2-5 October, 2017

University of Bergen, Bergen, Norway

${ }^{*}$ Speaker. 


\section{Introduction}

Since the first series of heavy ion experiments were carried out, high-energy nucleus-nucleus collisions have been an important tool to study QCD matter at high temperature. One of the most important findings is the existence of quark-gluon plasma (QGP), which behaves like a perfect fluid $[1,2,3,4,5,6,7]$. The hybrid framework of viscous hydrodynamics and hadronic cascade has been successful in describing a wide range of soft (low- $\left.p_{T}\right)$ observables in heavy ion collisions $[8,9$, $10,11]$. In addition to the bulk dynamics of QGP, measurements of the nuclear modification factor imply that jet-medium interactions are also useful probes [12, 13]. Even though many sophisticated studies have been done in both the hard and soft regimes, respectively, less attention has been paid to the intermediate $p_{T}$ ranges involved in heavy ion collision, where the thermal hadrons and minijet hadrons have comparable contributions. In this work, we extend a hybrid approach, which provides a reasonable description of the bulk dynamics, toward the higher $p_{T}$ range by having mini-jet production and energy loss. We also demonstrate that hadronic re-scatterings in the late stage of heavy ion collisions can significantly deform the momentum space distribution of final state hadrons.

\section{Model structure}

The initial state is determined by the IP-Glasma model $[14,11]$. The nucleon positions, from which one can find the positions of participants, spectators, and binary collisions, fluctuate event to event and determine the initial geometry of the collision. The pre-equilibrium dynamics are dominated by low-momentum gluons that are sourced by the hard partons in the colliding nuclei. The large occupation number of the small- $x$ gluons justifies treatment as a classical field. Thus, the gluon field is evolved in time using the classical Yang-Mills equations until $\tau_{0}=0.4 \mathrm{fm}$, at which the system is assumed to reach local thermal equilibrium.

The subsequent time evolution of the fireball is handled by means of the second-order viscous hydrodynamics, which is numerically solved by MUSIC [15]. Transport coefficients define how the system reacts to spatial anisotropy and inhomogeneity. In addition to shear and bulk viscosities, there are the second-order transport coefficients specifying contributions of the higher-order gradients $[16,17]$.

$$
\begin{aligned}
& \partial_{\mu} T^{\mu v}=0
\end{aligned}
$$

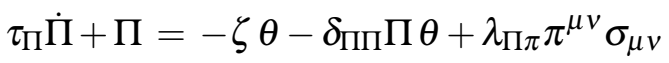

$$
\begin{aligned}
& \tau_{\pi} \dot{\pi}^{\langle\mu v\rangle}+\pi^{\mu v}=2 \eta \sigma^{\mu v}-\delta_{\pi \pi} \pi^{\mu v} \theta+\varphi_{7} \pi_{\alpha}^{\langle\mu} \pi^{v\rangle \alpha} \\
& -\tau_{\pi \pi} \pi_{\alpha}^{\langle\mu} \sigma^{v\rangle \alpha}+\lambda_{\pi \Pi} \Pi \sigma^{\mu v}
\end{aligned}
$$

where the temperature-dependent $\zeta / s$ has a peak at $T_{\text {peak }}=180 \mathrm{MeV}$ and $\eta / s$ is set to be 0.095 to reproduce the $p_{T}$-integrated flow coefficients $[8,11]$. The equations of motion for hydrodynamic system can be closed with the equation of state [18], which provides relations among energy density, charge densities, temperature and chemical potentials. In this work, vanishing baryon number density and baryonic chemical potential are assumed, a valid approximation in the mid-rapidity region of heavy ion collisions. 
Given that microscopic transport, implemented by means of an $N$-body simulation of hadrons, is necessary to describe non-equilibrium dynamics in the late stage of heavy ion collisions, the hydrodynamic information must be transformed into particles by a sampling procedure. This is done according to the Cooper-Frye formalism [19], which provides a connection between the momentum-space distribution and the 3-dimensional hypersurface where particles are emerging.

$$
\frac{d N}{d^{3} \mathbf{p}}=g \int_{\Sigma} f(x, \mathbf{p}) \frac{p^{\mu} d^{3} \Sigma_{\mu}}{E_{\mathbf{p}}}
$$

where the macroscopic information from hydrodynamics comes into the distribution function $f=$ $f_{0}+\delta f_{\text {shear }}+\delta f_{\text {bulk. }}$. In this work, the leading order contributions to $\delta f_{\text {shear }}$ and $\delta f_{\text {bulk }}$, which are respectively proportional to $\pi^{\mu v}$ [20] and $\Pi$ [21], are considered. Particles are sampled on the isothermal hypersurface with switching temperature $T_{\mathrm{sw}}=145 \mathrm{MeV}$, such that the proton $p_{T}$ spectrum is reproduced $[8,9]$.

To extend our hybrid approach toward the intermediate and higher $p_{T}$, jet production and energy loss must be involved in our framework. We perform jet production using PYTHIA 8 $[22,23]$ with a fixed minimum in transverse momentum transfer in $2 \rightarrow 2$ partonic interaction. The probability $P_{\text {hard }}$ that we have hard nucleon-nucleon process at each binary collision is given by

$$
\begin{aligned}
P_{\text {hard }}\left(\hat{p}_{T, \text { min }}\right) & =\frac{\sigma_{\text {hard }}\left(\hat{p}_{T, \text { min }}\right)}{\sigma_{\text {inel }}} \\
\sigma_{\text {hard }}\left(\hat{p}_{T, \text { min }}\right) & =\sum_{i, j} \int d x_{1} f_{i}\left(x_{1}\right) \int d x_{2} f_{j}\left(x_{2}\right) \hat{\sigma}_{i, j}\left(x_{1}, x_{2} ; \hat{p}_{T, \text { min }}\right)
\end{aligned}
$$

where $\hat{\sigma}_{i, j}$ and $\hat{p}_{T, \min }$ are respectively the partonic cross section and minimum transverse momentum transfer. $\hat{p}_{T, \min }$ is set to be $10 \mathrm{GeV}$ in the presented calculations. Mini-jet partons produced by hard interactions then propagate into thermal QGP medium and experience energy loss. There are two mechanisms that make jet partons lose energy — radiation of gluons [24] and collision with thermal partons [25]. Monte-Carlo implementation of energy loss with given hydrodynamic evolution is performed by MARTINI [26].

Hadronic cascade in the late stage of heavy ion collisions is handled by the UrQMD model $[27,28]$, which is microscopic transport of hadrons. It provides a solution to the transport equation

$$
p^{\mu} \frac{\partial f}{\partial x^{\mu}}=\mathscr{C}[f]
$$

by means of the $N$-body simulation, where individual particle can propagate and collide.

\section{Results}

We present the application of our hybrid approach for $\mathrm{Pb}-\mathrm{Pb}$ collisions at the LHC with $\sqrt{s_{N N}}=2.76 \mathrm{TeV}$. The shear and bulk viscosities and switching temperature are fixed such that experimental data for the integrated observables can be reproduced. Figure. 1 shows the identified hadrons $p_{T}$ spectra and $p_{T}$-differential elliptic flow of pions without MARTINI jets. From the functional form of the distribution function $f \sim e^{-p_{T} / T}$ at high $p_{T}$, it can be clearly seen that jet production and energy loss must be incorporated into our hybrid approach. Discrepancy in the 

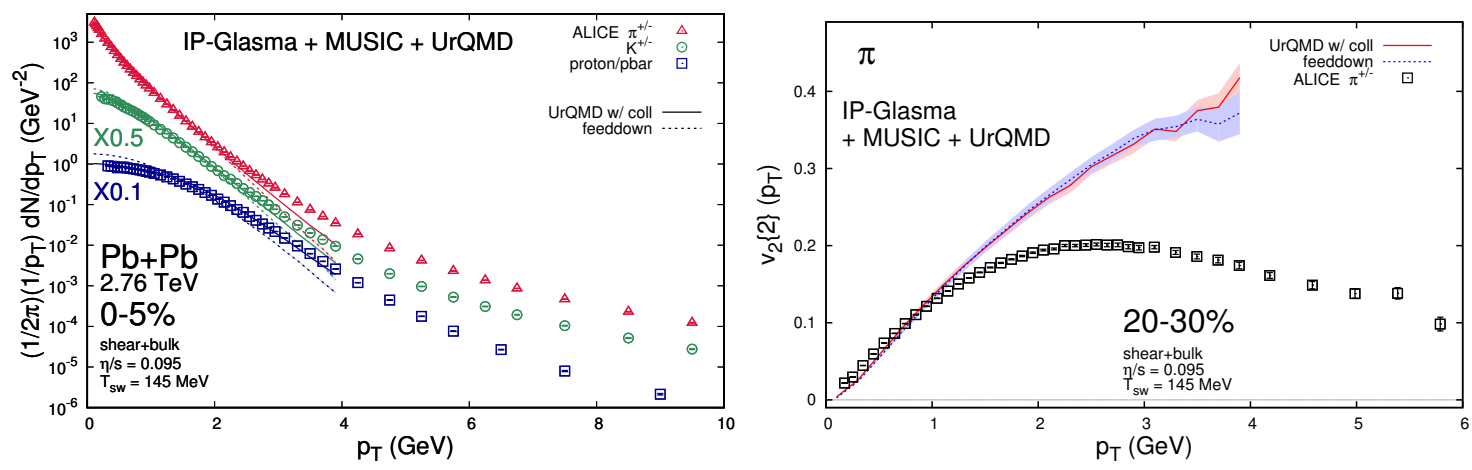

Figure 1: Identified hadrons $p_{T}$ spectra (left) and $p_{T}$-differential elliptic flow of pions (right) in the absence of jet production and energy-loss [9]. ALICE data are respectively from [29] and [30].
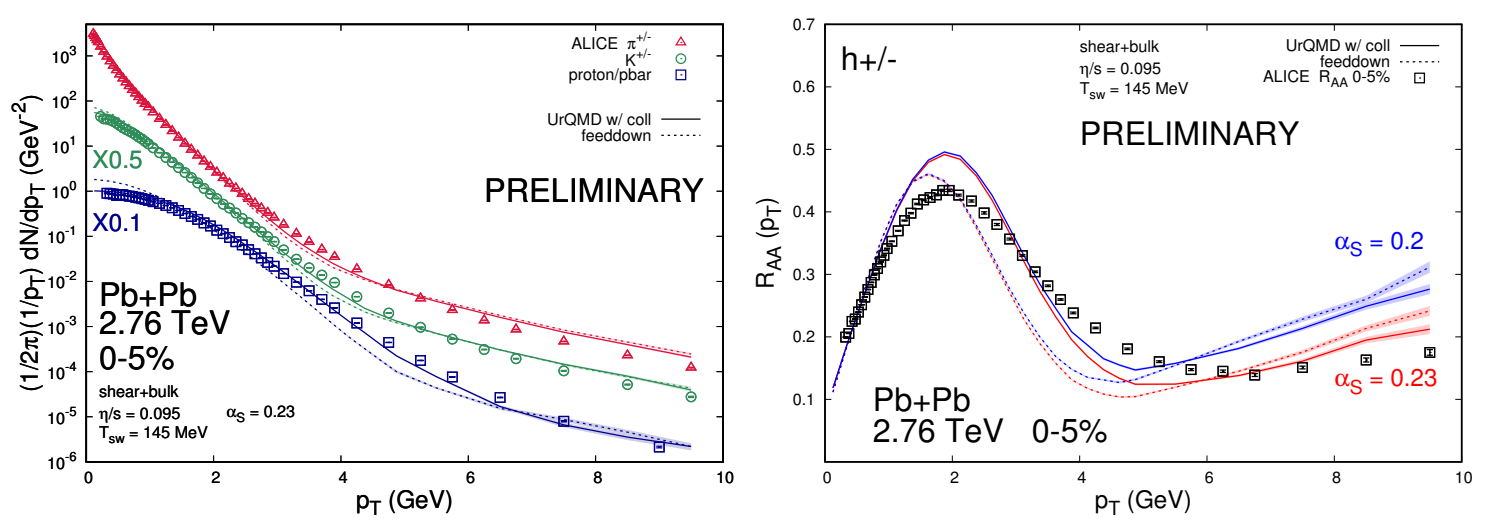

Figure 2: Identified hadrons $p_{T}$ spectra (left) and inclusive $R_{A A}$ of charged hadrons (right) in the presence of jet production and energy-loss. ALICE data are respectively from [29] and [31].

elliptic flow at intermediate and high $p_{T}$ is partially due to the fact that contributions of mini-jets with lower anisotropy are not taken into account.

As shown in Figure. 2, inclusion of mini-jet contributions enables us to describe the intermediate and higher $p_{T}$ observables within the hybrid approach. Hadronic re-scatterings have significant effects on baryonic spectra not only for the low $p_{T}$ range, but also for higher $p_{T}$. The nuclear modification factor $R_{A A}$ indicates that the effects of hadronic re-scatterings in the intermediate $p_{T}$ range are different from consequence of having different strong coupling $\alpha_{S}$ in the QGP phase. One can have an interpretation that mini-jet hadrons with high $p_{T}$ are decelerated while low- $p_{T}$ hadrons from the fireball are accelerated by re-scatterings.

Anisotropies in the momentum space are also affected by hadronic re-scattering, as depicted in Figure. 3. For pions and protons, re-scatterings result in more anisotropic particle distribution in the intermediate range $\left(3 \mathrm{GeV}<p_{T}<6 \mathrm{GeV}\right)$, where thermal and mini-jet hadrons have comparable contributions. Thermal hadrons, emerging from the fireball, are more anisotropic than mini-jet hadrons and shifted toward high- $p_{T}$ region by collisions with the jet hadrons. In addition, one can see that dependence of the radial and azimuthal distribution on hadronic cascade is stronger than 

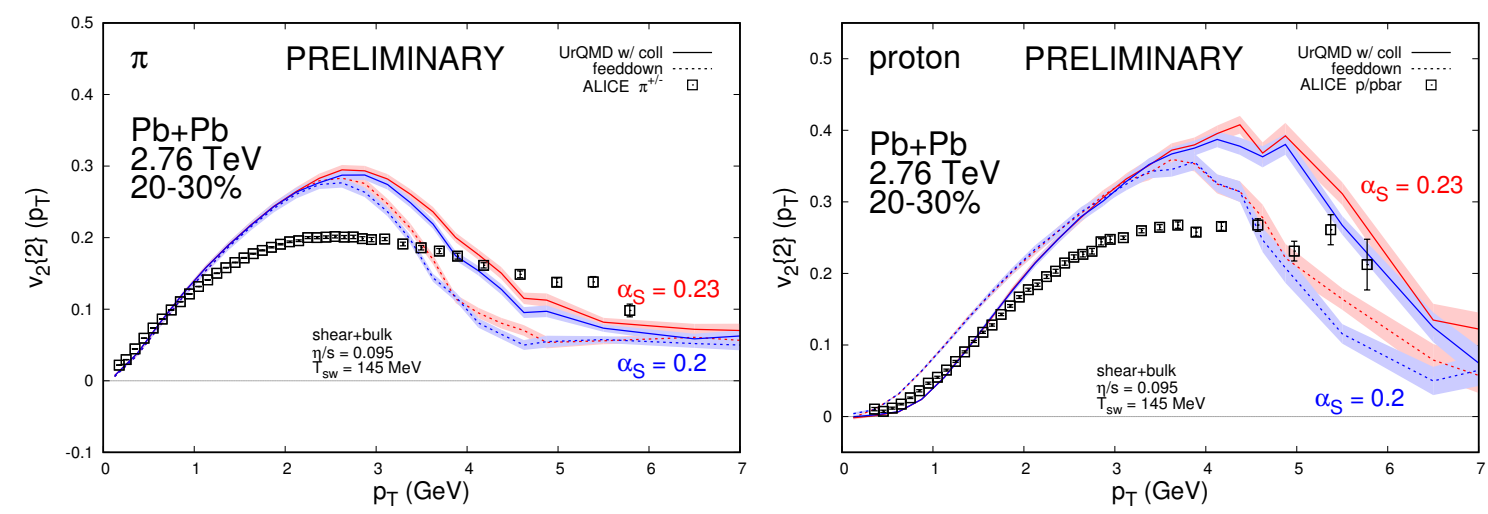

Figure 3: $p_{T}$-differential elliptic flow of pions (left) and protons (right) in the presence of jet production and energy-loss. ALICE data are from [30].

that on coupling constant in the QGP phase.

\section{Conclusion}

We model jet production and energy loss, combined with a hybrid model consisting of viscous hydrodynamics and hadronic afterburner for heavy ion collisions. Inclusion of the mini-jet contributions makes our hybrid approach capable of describing the intermediate and higher $p_{T}$ observables. We demonstrate that hadronic re-scatterings have distinct effects on the final state particle distribution. Thermal hadrons are accelerated by mini-jets and the $p_{T}$ spectra and anisotropic flow coefficients are shifted towards higher $p_{T}$. Those effects are unique in the sense that they cannot be compensated by different choice of strong coupling in the QGP phase. Given that deviation of the distribution function from the local thermal equilibrium becomes important in the intermediate $p_{T}$ range ( $3 \mathrm{GeV}<p_{T}<6 \mathrm{GeV}$ ), we lastly point out that a systematic study on the viscous correction $\delta f$ to the distribution function will improve the quality of description.

\section{Acknowledgements}

SR acknowledges support by the DFG through the grant CRC-TR 211. Computations were performed on the Guillimin supercomputer at McGill University under the auspices of Calcul Quebec and Compute Canada.

\section{References}

[1] BRAHMS collaboration, Nuclear Physics A 757, Issues 1-2, Pages 1-27 (2005).

[2] PHENIX collaboration, Nuclear Physics A 757, Issues 1-2, Pages 184-283 (2005).

[3] PHOBOS collaboration, Nuclear Physics A 757, Issues 1-2, Pages 28-101 (2005).

[4] STAR collaboration, Nuclear Physics A 757, Issues 1-2, Pages 102-183 (2005).

[5] C. Gale, S. Jeon and B. Schenke, Int. J. Mod. Phys. A 28, 1340011 (2013) 
[6] R. Derradi de Souza, T. Koide and T. Kodama, Prog. Part. Nucl. Phys. 86, 35 (2016)

[7] M. Gyulassy and L. McLerran, Nucl. Phys. A 750, 30 (2005).

[8] S. Ryu, J.-F. Paquet, C. Shen, G. S. Denicol, B. Schenke, S. Jeon and C. Gale, Phys. Rev. Lett. 115, no. $13,132301(2015)$

[9] S. Ryu, J. F. Paquet, C. Shen, G. Denicol, B. Schenke, S. Jeon and C. Gale, arXiv:1704.04216 [nucl-th].

[10] J. F. Paquet, C. Shen, G. S. Denicol, M. Luzum, B. Schenke, S. Jeon and C. Gale, Phys. Rev. C 93, no. 4, 044906 (2016)

[11] S. McDonald, C. Shen, F. Fillion-Gourdeau, S. Jeon and C. Gale, Phys. Rev. C 95, no. 6, 064913 (2017)

[12] K. M. Burke et al. [JET Collaboration], Phys. Rev. C 90, no. 1, 014909 (2014)

[13] S. Cao et al. [JETSCAPE Collaboration], Phys. Rev. C 96, no. 2, 024909 (2017)

[14] B. Schenke, P. Tribedy and R. Venugopalan, Phys. Rev. Lett. 108, 252301 (2012)

[15] B. Schenke, S. Jeon and C. Gale, Phys. Rev. C 82, 014903 (2010); Phys. Rev. Lett. 106, 042301 (2011); Phys. Rev. C 85, 024901 (2012).

[16] G. S. Denicol, S. Jeon and C. Gale, Phys. Rev. C 90, no. 2, 024912 (2014)

[17] G. S. Denicol, H. Niemi, E. Molnar and D. H. Rischke, Phys. Rev. D 85, 114047 (2012) Erratum: [Phys. Rev. D 91, no. 3, 039902 (2015)]

[18] P. Huovinen and P. Petreczky, Nucl. Phys. A 837, 26 (2010)

[19] F. Cooper and G. Frye, Phys. Rev. D 10, 186 (1974).

[20] K. Dusling, G. D. Moore and D. Teaney, Phys. Rev. C 81, 034907 (2010)

[21] P. Bozek, Phys. Rev. C 81, 034909 (2010)

[22] T. Sjöstrand, S. Mrenna and P. Z. Skands, JHEP 0605, 026 (2006)

[23] T. Sjöstrand, S. Mrenna and P. Z. Skands, Comput. Phys. Commun. 178, 852 (2008)

[24] P. B. Arnold, G. D. Moore and L. G. Yaffe, JHEP 0111, 057 (2001)

[25] G. Y. Qin, J. Ruppert, C. Gale, S. Jeon, G. D. Moore and M. G. Mustafa, Phys. Rev. Lett. 100, 072301 (2008)

[26] B. Schenke, C. Gale and S. Jeon, Phys. Rev. C 80, 054913 (2009)

[27] S. A. Bass et al., Prog. Part. Nucl. Phys. 41, 255 (1998)

[28] M. Bleicher et al., J. Phys. G 25, 1859 (1999)

[29] B. Abelev et al. [ALICE Collaboration], Phys. Rev. C 88, 044910 (2013)

[30] B. B. Abelev et al. [ALICE Collaboration], JHEP 1506, 190 (2015)

[31] K. Aamodt et al. [ALICE Collaboration], Phys. Lett. B 696, 30 (2011) 